\title{
Risk of acquired methemoglobinemia with different topical anesthetics during endoscopic procedures
}

This article was published in the following Dove Press journal:

Local and Regional Anesthesia

26 July 201 I

Number of times this article has been viewed

\author{
Srikanth Vallurupalli ${ }^{\prime}$ \\ Shalini Manchanda ${ }^{2}$ \\ 'Division of General Internal \\ Medicine, Department of Medicine, \\ Southern Illinois University School \\ of Medicine, Springfield, IL, USA; \\ ${ }^{2}$ Division of Pulmonary, Allergy, \\ Critical Care, and Occupational \\ Medicine, Department of Medicine, \\ Indiana University School of Medicine, \\ Indianapolis, IN, USA
}

Introduction: Methemoglobinemia is a recognized complication of the use of topical anesthetic sprays. The true scope of the problem or the risk with different topical anesthetic sprays and endoscopic procedures is unknown.

Methods: We retrospectively identified all cases of methemoglobinemia that occurred in a university affiliated community hospital from 2001 to 2007.

Results: Eleven cases of methemoglobinemia were identified over the 6-year period. Nine (82\%) occurred with use of benzocaine spray during transesophageal echocardiography (TEE). Patients who developed methemoglobinemia secondary to the topical anesthetic spray compared to other causes were more likely to be older, have lower mean hemoglobin levels $(10.5 \pm 0.5 \mathrm{~g} / \mathrm{dL}$ vs $11.3 \pm 0.0 \mathrm{~g} / \mathrm{dL})$, and a higher mean methemoglobin concentration at diagnosis $(40.8 \% \pm 5.2 \%$ vs $24 \% \pm 10 \%)$. However, only age reached statistical significance $(P=0.004)$.

Conclusion: In a university-affiliated community hospital, topical anesthetic sprays account for most of the burden of methemoglobinemia. Benzocaine use in the context of TEE caused more methemoglobinemia compared to lidocaine and other endoscopic procedures. This observation supports previous data and findings deserve further study.

Keywords: methemoglobinemia, benzocaine, lidocaine, transesophageal echocardiography, endoscopy

\section{Introduction}

Methemoglobin is an oxidized form of hemoglobin in which the heme iron exists in the ferric $\left(\mathrm{Fe}^{+3}\right)$ state. It is formed during the deoxygenation process and is reduced by various mechanisms including the cytochrome b5 reductase and nicotinamide adenine dinucleotide phosphate methemoglobin reductase pathways. ${ }^{1}$ The normal level in the serum is usually less than $1 \%$ and is kept in check by a balance between the processes that produce and reduce methemoglobin. An imbalance results in increased concentration leading to methemoglobinemia which results in impaired oxygen delivery to the tissues. Patients may present with shortness of breath, cough, dizziness, and cyanosis or may remain asymptomatic. The diagnosis is made by measuring methemoglobin levels using CO-oximetry in the arterial or venous blood. The causes may be congenital or acquired. Acquired causes include exposure to industrial chemicals, pesticides, medications such as dapsone and local anesthetics, and sepsis. ${ }^{2}$ A high degree of clinical suspicion is required for prompt diagnosis and treatment. There is increasing interest in the association of methemoglobinemia in the context of topical anesthetic use. Topical anesthetics are commonly used for oropharyngeal anesthesia during endoscopic procedures. The only published systematic review of
Correspondence: Srikanth Vallurupall Southern Illinois University School of Medicine, 70I N First Street, PO Box 19636, Springfield, IL 62794, USA

Tel +l 2175456914

$\mathrm{Fax}+\mathrm{I} 2175457127$

Email svallurupalli@siumed.edu 
methemoglobinemia in a hospital setting found that dapsone was responsible for $42 \%$ of all cases. ${ }^{2}$ Topical anesthesia (with $20 \%$ benzocaine spray) contributed to only $3.6 \%$ of cases of acquired methemoglobinemia. In the case report literature, benzocaine spray has been implicated in a majority of cases undergoing endoscopy. A recent review of literature indicated that methemoglobinemia was reported more frequently with use of benzocaine compared to lidocaine. ${ }^{3}$ There are however several limitations to relying on case report literature to draw conclusions regarding cause and effect. ${ }^{4}$ We undertook a systematic review of cases of acquired methemoglobinemia occurring in a university-affiliated community hospital with the following objectives: firstly to determine the contribution of local anesthetic use during endoscopy to the total burden of methemoglobinemia and secondly to determine the incidence of methemoglobinemia with use of different local anesthetics and endoscopic procedures.

\section{Materials and methods}

Carle Foundation Hospital is a community-based universityaffiliated tertiary care teaching hospital, designated a Level I trauma center in the Midwest of the US. The study was approved by the Institutional Review Board at the hospital and the University of Illinois at Urbana-Champaign. Cases of methemoglobinemia were obtained from the medical records from June 2001 to May 2007 using the International Classification of Diseases (ICD) 9 code 289.7. Acquired methemoglobinemia was defined as the presence of methemoglobin levels greater than $2 \%$. Data collected included age, sex, location of occurrence of methemoglobinemia, level of methemoglobin, and laboratory parameters including hemoglobin and glomerular filtration rate. The total number of endoscopic procedures done in the hospital during the same time period was determined using the hospital billing records. A 20\% benzocaine spray with $2 \%$ lidocaine gel at the tip of the endoscope is the standard local anesthetic used in the transesophageal echocardiography (TEE) lab while a 4\% lidocaine spray is used in the gastrointestinal endoscopy and bronchoscopy suites. Patients undergoing TEE also undergo a "swish and swallow" with $10 \mathrm{~mL}$ of $4 \%$ lidocaine solution. Patterns of anesthetic use were confirmed in consultation with physicians performing the procedures and pharmacy records. There were no fellows-in-training involved and all procedures were performed by trained and board-certified physicians in their respective fields. Statistical analysis was performed using SPSS software (v 16; IBM, New York, NY). Descriptive statistics and $t$-tests to explore differences in means were used for analysis of data.

\section{Results}

A total of eleven cases of acquired methemoglobinemia were identified. Nine of the eleven (82\%) occurred in the setting of topical anesthesia use, specifically with $20 \%$ benzocaine spray use during TEE. These cases were diagnosed in the endoscopy suite. The other two cases were diagnosed in the intensive care unit and were not related to endoscopy. One occurred in the context of severe sepsis and surgery related to a neck infection and one after endotracheal intubation in a patient with anaphylactic laryngeal edema (which did not involve topical anesthesia). Table 1 illustrates the number of different endoscopy procedures, the local anesthetic used, and the number of cases of methemoglobinemia encountered.

The average age of patients with methemoglobinemia was $62.6 \pm 3.8$ years and $64 \%$ were males. Four of these cases have been described in an earlier paper. ${ }^{11}$ Ten patients developed symptoms of shortness of breath and cyanosis. The diagnosis was suspected in one patient due to bluish color of his blood during a blood draw. The mean methemoglobin level at diagnosis was $37.5 \%$. Nine of the eleven patients had an active infection with bacteremia and one patient had osteomyelitis. All cases of bacteremia were secondary to Gram-positive cocci with Staphylococcus aureus being the most common agent $(\mathrm{n}=6,66 \%)$. All patients received methylene blue at doses of $1 \mathrm{mg} / \mathrm{kg}$ and recovered without major morbidity or mortality attributed to methemoglobinemia.

Patients who developed methemoglobinemia secondary to the topical anesthetic spray compared to other causes were more likely to be older (67.1 \pm 2.8 years vs $42.5 \pm 1.5$ years $)$, have lower mean hemoglobin levels $(10.5 \pm 0.5 \mathrm{~g} / \mathrm{dL}$ vs $11.3 \pm 0 \mathrm{~g} / \mathrm{dL}$ ), and a higher mean methemoglobin concentration at diagnosis $(40.8 \% \pm 5.2 \%$ vs $24 \% \pm 10 \%)$. However, only age was statistically significant $(P=0.004)$.

Table I Risk of methemoglobinemia with various endoscopic procedures and topical anesthetics

\begin{tabular}{llll}
\hline Endoscopic procedure & Topical anesthetic spray used & Number of procedures & Cases of methemoglobinemia \\
\hline Upper gastrointestinal endoscopy & $4 \%$ lidocaine spray & 21,767 & 0 \\
Bronchoscopy & $4 \%$ lidocaine spray & 443 & 0 \\
Transesophageal echocardiography & $20 \%$ benzocaine spray & 2,221 & $9(0.40 \%)$ \\
\hline
\end{tabular}


The incidence of congestive heart failure (28\% vs $0 \%)$, coronary artery disease (28\% vs $0 \%)$, and chronic renal insufficiency (19\% vs $0 \%$ ) was also higher in these patients.

\section{Discussion}

In this retrospective study, use of a $20 \%$ benzocaine spray was a common cause of acquired methemoglobinemia in a community based teaching hospital. This is in contrast to the study by Ash-Bernal and colleagues at Johns Hopkins University where benzocaine anesthesia accounted for only five of 138 cases. $^{2}$ The majority of cases in that series were related to the use of dapsone. This difference could be secondary to a difference in the patient population seen in these hospitals with different practice settings (inner city university hospital compared to a community hospital). The severity of illness and acuity of care provided may depend on geographic location, referral patterns, the presence of a transplant program, and a dedicated children's hospital. The prevalence of human immunodeficiency virus in the local population influences the use of drugs such as dapsone. The difference in study methodology may also be important. Methemoglobinemia was diagnosed through CO-oximetry data in the earlier study (with a methemoglobin level more than $2 \%$ considered diagnostic) while ours used hospital coding and billing data. Methemoglobinemia has a variable clinical presentation with a frequent lack of symptoms with lower levels of methemoglobin. This may have led to a failure to obtain the diagnostic test and resulted in underdiagnosis.

Benzocaine and lidocaine sprays are commonly used for topical oropharyngeal anesthesia during endoscopic procedures. Benzocaine belongs to the ester class of local anesthetics and is metabolized predominantly by plasma pseudocholinesterase. An N-hydroxy metabolite of benzocaine which contains an aniline group has been implicated in the oxidation of the ferrous ion resulting in increased methemoglobin production. ${ }^{5}$ Increased methemoglobin concentrations can occur even at appropriate doses. ${ }^{6}$ Genetic differences in metabolism may account for this variable effect of the drug. ${ }^{7}$ The spray formulation has been implicated in a majority of cases accounting for $93.2 \%$ of all cases in a review of data from the Adverse Event Reporting System of the Food and Drug Administration. ${ }^{8}$ This may be related to increased systemic absorption with the spray especially with inflamed or injured mucosa. Lidocaine is an amino amide class anesthetic. This difference in structure may explain the difference in their propensity to cause increased production of methemoglobin. Animal studies reveal an increased production of methemoglobin with benzocaine compared to lidocaine. ${ }^{7}$ In a recent review of the 242 cases reported in literature, benzocaine was implicated in $66 \%$ of all cases while lidocaine accounted for only about $5 \%{ }^{3}$ Based on available literature, several hospitals including the Veterans Health Administration have mandated the exclusive use of lidocaine sprays in their facilities. ${ }^{9}$ In the present study, all cases of methemoglobinemia occurred with use of benzocaine spray in the context of TEE. No cases occurred in the context of lidocaine spray use during other endoscopic procedures. This retrospective study suggests that lidocaine is less likely to cause methemoglobinemia than benzocaine when used during certain endoscopic procedures. However, this is confounded by the exclusive use of a particular agent in each of the endoscopy suites. The contribution of the lidocaine solution and gel applied to the tip of the TEE scope to the production of methemoglobin is also unknown. The notion that the type of endoscopic procedure itself influences the risk cannot be ruled out. It is interesting to note the relative lack of cases of methemoglobinemia during the course of a large number of upper gastrointestinal procedures over 6 years. The risk profile of patients undergoing these procedures may be different. A common indication for performing a TEE is the evaluation for endocarditis in patients with bacteremia. Infection results in increased oxidative stress and patients with sepsis have been noted to have higher methemoglobin levels. ${ }^{10}$ They also have a higher prevalence of several other risk factors for methemoglobinemia such as anemia, hypoalbuminemia, and the use of other offending drugs is also more prevalent. Patients with infection who undergo TEE with benzocaine are more likely to develop methemoglobinemia than those without an infection $(2.9 \%$ vs $0 \%, P<0.001) .{ }^{11}$ Transesophageal endoscopy is usually performed without fiber optic guidance (compared to gastrointestinal endoscopy and bronchoscopy) and thus may cause more mucosal trauma. This may lead to increased systemic absorption of the local anesthetic resulting in production of methemoglobin. The endoscopic procedure itself may thus influence the risk of methemoglobinemia.

\section{Limitations}

This was a retrospective study which relied on the use of ICD 9 codes for identification of cases. We were unable to procure $\mathrm{CO}$-oximetry data as employed by Ash-Bernal et al and had to rely on hospital records to identify cases of methemoglobinemia. ${ }^{2}$ All diagnosed cases of acquired methemoglobinemia may not have been 
captured due to improper coding. Patients may also have been underdiagnosed because of the nonspecific nature of symptoms especially in milder cases. There are no data on the dose of anesthetic administered though in the case of benzocaine, methemoglobinemia may occur with the use of appropriate dosage. We also did not study all the baseline characteristics of patients undergoing different procedures which may increase the risk of methemoglobin production. Awareness of this disease among different endoscopists was not studied. This may have influenced the number of cases diagnosed as clinical suspicion is essential in the diagnosis of this condition. The lack of a suitable control group (lidocaine use with TEE and benzocaine with other procedures) prevents us from drawing any concrete conclusions regarding the increased propensity of methemoglobin formation with benzocaine.

\section{Conclusion}

Topical anesthetic use with $20 \%$ benzocaine caused a majority of cases of acquired methemoglobinemia in a university-affiliated community hospital. This knowledge can be used to enhance patient safety by deploying educational and diagnostic resources to areas of the hospital that routinely use these agents. This study illustrates the increased occurrence of methemoglobinemia in the setting of active infection. Clinicians should consider methemoglobinemia as a cause of respiratory difficulty in infected patients exposed to topical anesthetics. The study also supports case report data that benzocaine use may result in more cases of methemoglobinemia than lidocaine. This should be confirmed in a prospective controlled study. Until such studies are performed, lidocaine may be a safer alternative especially in patients with an active infection. The finding of increased incidence with TEE compared to other endoscopic procedures may be related to the anesthetic used, but deserves further study.

\section{Acknowledgments}

The authors would like to thank Tricia Truscott, Health Information Management, Carle Foundation Hospital, Urbana, IL for assisting with data collection and Dr Andrea $\mathrm{V}$ Brasch for help with interpreting the data.

\section{Disclosure}

The authors report no conflicts of interest in this work.

\section{References}

1. Umbreit J. Methemoglobin - it's not just blue: a concise review. Am J Hematol. 2007;82(2):134-144.

2. Ash-Bernal R, Wise R, Wright SM. Acquired methemoglobinemia: a retrospective series of 138 cases at 2 teaching hospitals. Medicine (Baltimore). 2004;83(5):265-273.

3. Guay J. Methemoglobinemia related to local anesthetics: a summary of 242 episodes. Anesth Analg. 2009;108(3):837-845.

4. Doherty M. What value case reports? Ann Rheum Dis. 1994;53(1): $1-2$.

5. Clary B, Skaryak L, Tedder M, Hilton A, Botz G, Harpole D. Methemoglobinemia complicating topical anesthesia during bronchoscopic procedures. J Thorac Cardiovasc Surg. 1997;114(2):293-295.

6. Guertler AT, Pearce WA. A prospective evaluation of benzocaineassociated methemoglobinemia in human beings. Ann Emerg Med. 1994;24(4):626-630.

7. Guertler AT, Lagutchik MS, Martin DG. Topical anesthetic-induced methemoglobinemia in sheep: a comparison of benzocaine and lidocaine. Fundam Appl Toxicol. 1992;18(2):294-298.

8. Moore TJ, Walsh CS, Cohen MR. Reported adverse event cases of methemoglobinemia associated with benzocaine products. Arch Intern Med. 2004;164(11):1192-1196.

9. Moos DD, Cuddeford JD. Methemoglobinemia and benzocaine. Gastroenterol Nurs. 2007;30(5):342-345.

10. Ohashi K, Yukioka H, Hayashi M, Asada A. Elevated methemoglobin in patients with sepsis. Acta Anaesthesiol Scand. 1998;42(6):713-716.

11. Vallurupalli S, Das S, Manchanda S. Infection and the risk of topical anesthetic induced clinically significant methemoglobinemia after transesophageal echocardiography. Echocardiography. 2010;27(3): 318-323.
Local and Regional Anesthesia

\section{Publish your work in this journal}

Local and Regional Anesthesia is an international, peer-reviewed, open access journal publishing on the development, pharmacology, delivery and targeting and clinical use of local and regional anesthetics and analgesics. The journal welcomes submitted papers covering original research, basic science, clinical studies, reviews \& evaluations,

\section{Dovepress}

guidelines, expert opinion and commentary, case reports and extended reports. The manuscript management system is completely online and includes a very quick and fair peer-review system, which is all easy to use. Visit http://www.dovepress.com/testimonials.php to read real quotes from published authors. 